\title{
»Eine Geschichte vom Zweifel und vom Glauben«.
}

\author{
Dietrich Bonhoeffer und Zweifel
}

Christine Schliesser

"Eine Geschichte vom Zweifel und vom Glauben «. ${ }^{1}$ So beschreibt Dietrich Bonhoeffer die Geschichte Gideons in seiner Predigt zu Richter 6,15f. aus dem Jahr 1933. Es könnte zugleich auch eine Überschrift über Bonhoeffers eigenes Leben sein, sah er sich doch als einen, der "glauben lernen" wollte. ${ }^{2}$ Dass ihm dabei Zweifel verschiedenster Art begegnen würden, war für ihn weder Grund zur Verwunderung noch Anlass zur Besorgnis. Er konnte Zweifel als einen wesentlichen und notwendigen Bestandteil dieses Lernprozesses sehen, der letztlich der Glaubensstärkung dient. ${ }^{3}$

Es ist nicht bekannt, dass sich Bonhoeffer einmal in seinem fragmentarisch gebliebenen Werk in einem größeren Zusammenhang systematisch-theologisch mit dem Phänomen des Zweifels auseinandergesetzt hat. Und doch tritt es punktuell an verschiedenen Stellen und in ganz unterschiedlichen Kontexten wie Predigten, Bibelarbeiten, Vorlesungen oder Briefen hervor und ermöglicht uns so einen Einblick in seine Gedanken. Im Folgenden soll daher versucht werden, aus zweierlei Blickwinkeln diese Gedanken $\mathrm{zu}$ sammeln und nachzuzeichnen. Es geht dabei zum einen um Selbstzweifel, die sich bei Bonhoeffer als Zweifel über ganz konkrete und praktische Entscheidungen bis hin zu existentiellen Zweifeln an der eigenen Person manifestieren. Da sie bei Bonhoeffer vor allem biographisch verankert sind und uns damit Bonhoeffer auch

1 Predigt zu Richter 6,15f.; 7,2; 8,23, Berlin, Estohmihi, 26.02.1933, in: Dietrich Bonhoeffer, Berlin 1932-1933, Dietrich Bonhoeffer Werke (DBW) 12, hg. v. C. Nicolaisen und E.-A. Scharffenorth, Gütersloh 1997, 447-454, 448.

2 Vgl. Bonhoeffers Charakterisierung Gideons als einen Mann, der "glauben gelernt hat.«, Predigt zu Richter 6,15f.; 7,2; 8,23, in: Bonhoeffer, Berlin, 450. Bekannter ist Bonhoeffers Brief aus der Haft an seinen Freund Eberhard Bethge, in dem er davon berichtet, dass auch er "glauben lernen« wollte. Dietrich Bonhoeffer, Widerstand und Ergebung, Briefe und Aufzeichnungen aus der Haft, DBW 8, hg. v. Ch. Gremmels et al., Gütersloh 1998, 542.

3 Vgl. Bonhoeffers Konfirmationspredigt zu Markus 9,24 ("Ich glaube, lieber Herr, hilf meinem Unglauben«), Konfirmation, Kieckow, 09.04.1938, in: Dietrich Bonhoeffer, Illegale Theologenausbildung: Sammelvikariate 1937-1940, DBW 15, hg. v. Dirk Schulz, Gütersloh 1998, 476-481, 480. 
als Person näher bringen, soll mit diesen begonnen werden. Anschließend beschäftige ich mich mit Bonhoeffers theologischer Auseinandersetzung mit Zweifel, insbesondere als Gotteszweifel. Bonhoeffer behandelt diesen vor allem im Zusammenhang von Anfechtung und Versuchung sowie im Verhältnis Zweifel und Glaube. Dass beides, Selbst- und Gotteszweifel, auch eng miteinander verwoben sein kann, soll durch diese Aufteilung nicht in Abrede gestellt werden. Im Gegenteil, das bisweilen enge Aufeinanderbezogensein von Selbst- und Gotteszweifel wird deutlich sichtbar werden.

\section{Selbstzweifel}

\section{1 »Zweifel am eigenen Weg « ${ }^{4}$}

Auch wenn Bonhoeffers souveränes und selbstsichereres Auftreten es in der Regel gut verbarg, waren ihm Selbstzweifel durchaus nicht fremd. So kannte er das ernste und bisweilen erbitterte Ringen um die rechte Entscheidung gut. Selbst wenn er eine Entscheidung schließlich gefällt hatte, konnten ihn die Zweifel über die Richtigkeit noch lange belasten und gegebenenfalls sogar wieder umstimmen. Beispielhaft sei hier sein innerer Kampf erwähnt, als er 1939 die Möglichkeit hatte, mit Hilfe amerikanischer Freunde das kriegsbereite Nazi-Deutschland zu verlassen und sich in den Vereinigten Staaten in Sicherheit zu bringen. Dort angekommen, plagten ihn die "Zweifel am eigenen Weg « ${ }^{5}$ jedoch so sehr, und "die ganze Wucht der Selbstvorwürfe wegen einer Fehlentscheidung « ${ }^{6}$ drohte ihn fast zu erdrücken, so dass er schließlich mit dem letzten Schiff vor Kriegsausbruch nach Deutschland zurückkehrte. In dem Versuch, die bestimmenden Faktoren in seinen Entscheidungsprozessen zu entschlüsseln, kam er zu folgender Einsicht: »Es ist merkwürdig, ich bin mir bei allen meinen Entscheidungen über die Motive nie völlig klar. [...] Man kann eben alles begründen. Zuletzt handelt man doch

\footnotetext{
E. Bethge, Dietrich Bonhoeffer, Eine Biographie, Gütersloh ${ }^{8} 1994,731$.

Vgl. seinen Tagebucheintrag einen Tag vor der Landung in New York, 11. Juni 1939: "Wenn nur die Zweifel am eigenen Weg überwunden wären. ", Tagebuch der Amerikareise, 08.06.-09.07.1939, in: Bonhoeffer, Sammelvikariate, 217-240, 219.

6 Tagebucheintrag 15. Juni 1939, Tagebuch der Amerikareise, 08.06.-09.07.1939, in: Bonhoeffer, Sammelvikariate, 223.
} 
aus einer Ebene heraus, die uns verborgen bleibt. Darum kann man nur bitten, dass Gott uns richten und vergeben wolle. « ${ }^{7}$

\section{$1.2 »$ Wer bin ich? $«^{8}$}

Neben den Zweifeln am eigenen Weg und dem Ringen um die rechte Entscheidung sah sich Bonhoeffer, vor allem in seinen letzten Lebensjahren, mit noch einer anderen, möglicherweise noch tiefer gehenden Art von Selbstzweifeln konfrontiert: Nun sind nicht mehr nur einzelne Entscheidungen im Zentrum des Zweifels, sondern die eigene Person, das eigene Ich wird in Frage gestellt. In seinem während der Haftzeit entstandenen Gedicht "Wer bin ich?« versucht Bonhoeffer, diese fundamentalen Zweifel am eigenen Selbst zum Ausdruck zu bringen und $\mathrm{zu}$ verarbeiten. Das Gedicht ist so eindrücklich, dass es für sich selbst sprechen soll. Im Folgenden sei es daher in Auszügen zitiert:

Wer bin ich?

Wer bin ich? Sie sagen mir oft,

ich träte aus meiner Zelle

gelassen und heiter und fest,

wie ein Gutsherr aus seinem Schloss.

Bin ich das wirklich, was andere von mir sagen? oder bin ich nur das, was ich selbst von mir weiß? unruhig, sehnsüchtig, krank, wie ein Vogel im Käfig, ringend nach Lebensatem, als würgte mir einer die Kehle

müde und leer zum Beten, zum Denken, zum Schaffen, matt und bereit, von allem Abschied zu nehmen?

Wer bin ich? Einsames Fragen treibt mit mir Spott.

Wer ich auch bin, Du kennst mich, Dein bin ich, o Gott!

\footnotetext{
7 Tagebucheintrag 20.Juni 1939, Tagebuch der Amerikareise, 08.06.-09.07.1939, in: Bonhoeffer, Sammelvikariate, 228.

8 So der Titel eines bekannten Gedichts Bonhoeffers, verfasst in den Tagen vor dem erwarteten Umsturz im Juli 1944, Bonhoeffer, Widerstand und Ergebung, $513 f$.
} 


\section{Gotteszweifel}

\subsection{Zweifel als Versuchung und Anfechtung}

Nach der biographisch-existentiellen Dimension des Zweifels wie sie Bonhoeffer als Selbstzweifel erfuhr, soll es nun um seine theologische Auseinandersetzung mit dem gehen, was hier unter dem Begriff Gotteszweifel zusammengefasst wird. Wenn Bonhoeffer von "Zweifel

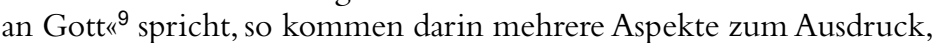
die inhaltlich teilweise auch ineinander übergehen, so u.a. Zweifel an Gottes Geboten, seinerVergebung und Liebe, Zweifel als Versuchung und Anfechtung oder Zweifel in Relation zu Glaube. Am ausführlichsten setzte sich Bonhoeffer mit Gotteszweifel im Kontext einer längeren Bibelarbeit über die sechste Bitte desVaterunsers (»Führe uns nicht in Versuchung, sondern erlöse uns von dem Übel.« Matthäus 6,13) für seine Seminaristen im Sommer 1938 auseinander. ${ }^{10}$

In der Versuchung als "konkretes, aus dem Verlauf des Lebens jäh heraustretendes Ereignis «11 überfällt der Zweifel als plötzliche Ungewissheit, Sinnlosigkeit und Traurigkeit mit Macht den Menschen. Das gesamte Ich gerät ins Wanken. Auch hier zeigt sich, wie eng Gotteszweifel und Selbstzweifel verflochten sein können. Analog zu den beiden biblischen Versuchungsgeschichten gibt es für Bonhoeffer zwei mögliche Interpretationszusammenhänge der Versuchung: Entweder die Versuchung gilt dem Adam in uns und bringt uns damit zu Fall oder aber sie gilt Christus in uns, der uns zu widerstehen hilft. Denn wie in der Versuchung Adams alle gefallen sind, gilt allen der Sieg, den Jesus Christus in seiner Versuchung errungen hat. Doch wo hat die Versuchung, die sich als Zweifel an Gott manifestiert, ihren Ursprung? Bonhoeffer weist auf drei verschiedene Urheber hin: Zum einen den Teufel als »zum Zweifel an Gottes Wort und zum Abfall von Gott « ${ }^{12}$ Verführenden und als Lügner: "Der Zweifel an Gottes Wort ist die Lüge schlechthin«. ${ }^{13}$

\footnotetext{
9 Vgl. Bibelarbeit über Versuchung, 20.-25.06.1938, in: Bonhoeffer, Sammelvikariate, 371-406, 380 .

${ }^{10}$ Bibelarbeit über Versuchung, 20.-25.06.1938, in: Bonhoeffer, Sammelvikariate, 371-406.

${ }^{11}$ Bibelarbeit über Versuchung, 20.-25.06.1938, in: Bonhoeffer, Sammelvikariate, 373.

${ }^{12}$ Bibelarbeit über Versuchung, 20.-25.06.1938, in: Bonhoeffer, Sammelvikariate, 384.

${ }^{13}$ Bibelarbeit über Versuchung, 20.-25.06.1938, in: Bonhoeffer, Sammelvikariate, 385, Fußnote 46.
} 
Als zweites nennt Bonhoeffer die menschliche Begierde als Lust und Leidensscheu. Damit schließt er zugleich jede Selbstrechtfertigung aus, die den Ursprung des Zweifels gänzlich jenseits der eigenen Person verorten möchte. Und schließlich ist auch Gott als Ursprung der Versuchung genannt. Am Beispiel der Hiobsgeschichte macht Bonhoeffer deutlich, dass nichts ohne Gottes Wissen und Willen geschieht. An der Gestalt Hiobs wird aufgezeigt, dass der Mensch zwar durchaus gegen das Leiden und - wie Luther beim Tod seiner Tochter Magdalene ${ }^{14}$ - gegen das Einbrechen des Teufels in Gottes Ordnungen murren darf. Und doch muss der Mensch sich nach Bonhoeffer bewusst bleiben, dass sein Leiden unter der Perspektive des Gerichtes Gottes angesichts der Sündhaftigkeit des Menschen stets gerechtfertigt ist.

Als besondere Formen derVersuchung kennt Bonhoeffer die securitas, den geistlichen Hochmut, und die desperatio, die Traurigkeit, mit ihren jeweiligen verschiedenen Ausprägungen. Vor allem Letztere sät Zweifel an Gottes Gegenwart, Güte und Vergebung, die so überhand nehmen können, dass sie zur Versuchung zur "furchtbaren Sünde ${ }^{15}$ werden können. Bonhoeffer selbst wusste um seine Gefährdung diesbezüglich, wie er in einem Brief seinem Freund Eberhard Bethge eingestand: »Du bist der einzige Mensch, der weiß, dass die racedia - tristitia mit ihren bedrohlichen Folgen mir oft nachgestellt hat, und hast Dir vielleicht - so fürchtete ich damals - in dieser Hinsicht Sorgen um mich gemacht. Aber ich habe mir von Anfang an gesagt, dass ich weder den Menschen noch dem Teufel diesen Gefallen tun werde; dies Geschäft sollen sie selbst besorgen, wenn sie wollen; und ich hoffe, immer dabei bleiben zu können. «16

Einige theologische Fragezeichen bleiben freilich bestehen in Bonhoeffers Bibelarbeit über die Versuchung. So gelingt es ihm nicht immer überzeugend, die Spannungsfelder zwischen der "Hand des Teufels" und der "Hand Gottes ${ }^{17}$ aufzulösen. Auch führt die Aneinanderreihung verschiedenster Bibelstellen, teilweise gänzlich ohne Exegese, nicht immer zur gewünschten Klarheit. Kennzeichnend für diese Bibelarbeit ist daher auch weniger ihre systematisch-theologische Stringenz als vielmehr der Versuch, die

${ }^{14}$ Vgl. Luthers Brief an Justus Jonas vom 23.09.1542, M. Luther, WA.BR 10, $149 \mathrm{f}$.

15 Bibelarbeit über Versuchung, 20.-25.06.1938, in: Bonhoeffer, Sammelvikariate, 374.

${ }^{16}$ Bonhoeffer, Widerstand und Ergebung, 187.Vgl. auch Bonhoeffer zur "Versuchung zum Selbstmord «, Dietrich Bonhoeffer, Ethik, DBW 6, hg. v. I. Tödt et al., München 1992, 199.

${ }^{17}$ Bibelarbeit über Versuchung, 20.-25.06.1938, in: Bonhoeffer, Sammelvikariate, 390 . 
angehenden Pfarrerinnen und Pfarrern der illegalen Bekennenden Kirche in ihren ganz realenVersuchungen, Ängsten und Zweifel ernst $\mathrm{zu}$ nehmen und auf Grundlage biblischer Versuchungsgeschichten $\mathrm{zu}$ ermutigen und $\mathrm{zu}$ bestärken. Auch in diesem Sinne verweist Bonhoeffer abschließend in seiner Bibelarbeit auf Luthers bekanntes Diktum: »Keine Anfechtung haben ist die schwerste Anfechtung. «18

In eine ähnliche Richtung weisen Bonhoeffers Überlegungen in seiner Predigt zu Matthäus 8,23ff (»Die Stillung des Sturmes") aus dem Jahr 1933. Wenn die Anfechtung überhand zu nehmen scheint und die Zweifel an Gottes barmherziger Gegenwart in Verzweiflung umzuschlagen drohen, dann sollen "wir doch die Stunden der Heimsuchung und Anfechtung, die Stunden auf hohem Meer in unserem Leben so recht verstehen [...] Gott ist ihnen nah, nicht fern «. ${ }^{19}$ Der Grund dafür lautet: »Unser Gott ist im Kreuz. ${ }^{20}$ Damit klingt hier bereits ein Gedanke an, der Bonhoeffer bis in seine Gefängnisjahre beschäftigen sollte. Weil Christus alle Anfechtung und Versuchung durchlitten und überwunden hat, sind in ihm auch unsere Anfechtung und Versuchung besiegt. Doch geht der Sieg durchs Kreuz, sowohl für Christus als auch für seine Nachfolger: "Christus ist der Gekreuzigte und ungekreuzigt kommt kein Christ zum Leben. ${ }^{21}$ Aus dieser Kreuzestheologie, die das Leiden Christi und das Mitleiden seiner Nachfolger betont, wird Bonhoeffer in den schwersten Stunden seines Lebens die Kraft zum Aushalten seiner eigenen Zweifel und seines eigenen Leides zu ziehen suchen: »Nur der leidende Gott kann helfen. ${ }^{22}$

Entsprechendes galt auch für Luther, bei Bonhoeffer die meistzitierte Autorität: Gott ist nicht zu finden, "nisi in passionibus et cruce $\ll^{23}$. Die Anfechtung und der ihr innewohnende Zweifel müssen daher recht gedeutet werden: nicht als unser Verlassensein von Gott, sondern ganz im Gegenteil als besondere Nähe des Gottes, der das Angefochtensein kennt und überwand. In sei-

\footnotetext{
${ }^{18}$ Bibelarbeit über Versuchung, 20.-25.06.1938, in: Bonhoeffer, Sammelvikariate, 403, Fußnote 98.

19 Predigt zu Matthäus 8,23-27, Berlin, 2. Sonntag nach Epiphanias, 15.01.1933, in: Bonhoeffer, Berlin, 439-447, 445.

${ }^{20}$ Predigt zu Matthäus 8,23-27, Berlin, 2. Sonntag nach Epiphanias, 15.01.1933, in: Bonhoeffer, Berlin, 445

${ }^{21}$ Predigt zu Matthäus 8,23-27. Berlin, 2. Sonntag nach Epiphanias, 15.01.1933, in: Bonhoeffer, Berlin, 446

${ }^{22}$ Bonhoeffer, Widerstand und Ergebung, 534.

23 "At Deum non inveniri nisi in passionibus et cruce, iam dictum est." ("Aber es ist schon gesagt, dass Gott nur in Leiden und Kreuz zu finden ist."), M. Luther, WA 1, 362, 28f. (These XXI. Disputatio Heidelbergae habita, 1518).
} 
ner Christologievorlesung aus dem Sommersemester 1933 kann Bonhoeffer der Anfechtung daher auch durchaus positive Seiten abgewinnen, lehrt sie doch, auf Gottes Wort zu achten. Und schließlich gilt sogar: »Aus der Anfechtung kommt der Glaube. ${ }^{24}$

Damit ist ein weiteres Themenfeld angerissen, das im Kontext des Gotteszweifels bedacht werden muss: Das Verhältnis von Zweifel und Glaube.

\subsection{Zweifel und Glaube}

Für Bonhoeffer kann über Gotteszweifel nicht abseits des Glaubens geredet werden, wird der Zweifel doch erst verständlich auf dessen Hintergrund. Dabei ist es besonders auffällig, dass Bonhoeffer Zweifel nicht einfach als Gegensatz von Glauben deutet. In seinen Predigten ist es ihm daher immer wieder ein Anliegen, dem Gotteszweifel seine vermeintliche Sinnlosigkeit zu nehmen und dessen glaubensstärkende Funktion herauszustellen. So stellt er in der eingangs erwähnten Predigt über Gideon aus dem Jahr 1933 einen engen Zusammenhang, geradezu eine notwendige Wechselwirkung zwischen Glaube und Zweifel her. Die Geschichte des Gideon als einem der "Männer des Glaubens ${ }^{25}$ ist nicht etwa linear zu denken, als würde er schließlich und ein für alle Mal vom Zweifel zum Glauben durchbrechen. Stattdessen sind Zweifel und Glauben eng miteinander verwoben, wo Glaube ist, ist der Zweifel nicht weit. Die biblischen "Männer des Glaubens» sind daher immer zugleich "Zweifler«; Gideon ist kein "Siegfried«, sondern verkörpert ein "menschlich unschönes, unharmonisches, zerfetztes Menschenbild. ${ }^{26}$ Aber dennoch und auch gerade deshalb das "Bild des Menschen, der glauben gelernt hat. «27

Das Motiv des Zweifels, der der Glaubensstärkung dient, findet sich auch in einer Konfirmationspredigt aus dem Jahr 1938 zu Markus 9,24 (»Ich glaube, hilf meinem Unglauben«). Dort bereitet Bonhoeffer die Konfirmanden darauf vor, dass der Zweifel an Gott und seinen Geboten mit Sicherheit über sie hereinbrechen wird. Zugleich betont er aber, dass die Erfahrungen des Zweifels für die Lebendigkeit und die Stärkung des Glaubens notwendig seien: »Das muss alles so kommen, so gewiss euer Glaube lebendig ist. Das muss

\footnotetext{
${ }^{24}$ Vorlesung "Christologie» (Nachschrift), Sommersemester 1933, in: Bonhoeffer, Berlin, 279-348, 346.

${ }_{25}$ Predigt zu Richter 6,15f.; 7,2; 8,23, in: Bonhoeffer, Berlin, 450.

${ }^{26}$ Predigt zu Richter 6,15f.; 7,2; 8,23, in: Bonhoeffer, Berlin, 448, 450.

${ }^{27}$ Predigt zu Richter 6,15f.; 7,2; 8,23, in: Bonhoeffer, Berlin, 450.
} 
alles so kommen, damit euer Glaube geprüft und gestärkt wird. «28 Wiederum beschreibt er das Verhältnis von Glaube und Zweifel als ein dynamisches und wendet sich gegen einen Glaubensbegriff, der den Glauben in etwas stets Verfügbares umdeuten möchte. Im Gegensatz dazu vergleicht er den Glauben mit dem Manna, das das Volk Israel nie auf Vorrat, sondern stets jeden Tag aufs Neue empfing: "So ist es auch mit dem Glauben. Entweder wir empfangen ihn täglich neu, oder er wird faul. [...] Es ist jeden Morgen ein neuer Kampf durch allen Unglauben, durch allen Kleinglauben, durch alle Unklarheit und Verworrenheit, durch alle Furchtsamkeit und Ungewissheit zum Glauben hindurchzustoßen und ihn Gott abzuringen. ${ }^{29}$

Ein neuer Aspekt tritt in Bonhoeffers Predigtmeditation $\mathrm{zu}$ Johannes 20,19-31 aus dem Jahr 1940 über Thomas, "den Zweifler«, hinzu. Dort spricht Bonhoeffer von einem "aufrechten" Zweifel. Auch hier wird der Zweifel dem Glauben nicht schlicht gegenüber, sondern eher in eine Beziehung zu ihm gestellt.Auch wenn »der zweifelnde Thomas" geradezu zu einer Personifizierung des Zweifels an Gott geworden ist, bescheinigt ihm Bonhoeffer die "Bereitwilligkeit $[\ldots]$, sich überzeugen zu lassen" und die "Aufrichtigkeit seines Zweifels ${ }^{30}{ }^{30}$ Es gibt also eine Art von Zweifel, die sich nicht in sich selbst verschließt, sondern auf das Überzeugtwerden und damit auf den Glauben hin ausgerichtet ist. Dennoch ist es letztlich freilich nicht der Zweifel, sondern allein der Glaube, den Jesus in seiner Antwort zu Thomas selig preist.

Der Hinweis, dass Thomas mit seinen Zweifeln nicht die Ausnahme ist und dass "Millionen von Zweiflern [...] nach Thomas kommen « werden ${ }^{31}$, scheint Bonhoeffers eigene, spätere Erfahrung vorweg zu nehmen, dass der Glaube nicht etwas Statisches ist, sondern etwas Dynamisches, das angefochten werden kann. So macht er im Gefängnis die Erfahrung, dass »einem ganz ohne ersichtlichen Grund manche Tage so viel schwerer werden als andere " und äußert dieVermutung, dass »es Anfechtungen [sind] «. ${ }^{32}$ Doch ebenso kennt er Tage, an denen jeglicher Zweifel fern bleiben muss: „Gottes Hand und Führung ist mir so gewiss, dass ich hoffe, immer in dieser Gewissheit bewahrt zu werden. «33

\footnotetext{
${ }^{28}$ Konfirmationspredigt zu Markus 9,24, in: Bonhoeffer, Sammelvikariate, 480.

${ }^{29}$ Konfirmationspredigt zu Markus 9,24, in: Bonhoeffer, Sammelvikariate, 478.

${ }^{30}$ Predigtmeditation zu Johannes 20,19-31, Für den Sonntag Quasimodogeniti,1940, in: Bonhoeffer, Sammelvikariate, 554-559, 558.

${ }^{31}$ Predigtmeditation zu Johannes 20,19-31, in: Bonhoeffer, Sammelvikariate, 559.

32 Bonhoeffer, Widerstand und Ergebung, 399.

${ }^{33}$ Bonhoeffer, Widerstand und Ergebung, 576.
} 


\section{Schlussbetrachtung: Christus als Überwinder des Zweifels}

So unterschiedlich die Kontexte sind, aus denen Bonhoeffers Beschäftigung mit dem Zweifel hervorgegangen ist, und so verschieden die jeweiligen Schwerpunkte, so gibt es dennoch eine auffällige Gemeinsamkeit, die gleich einem roten Faden die Überlegungen untereinander verknüpft: Bonhoeffers Aussagen über den Zweifel ist gemein, dass er versucht, die damit verbundenen Probleme christologisch zu lösen, ein Merkmal seines oft als "christozentrisch " ${ }^{34}$ beschriebenen theologischen Ansatzes. Jesus Christus ruft den Menschen aus seinem Zweifel, aus seiner Zwiespältigkeit heraus. An den Menschen ergeht der "Ruf aus der Entzweiung, aus dem Abfall, aus dem Wissen um Gut und Böse heraus, zur Versöhnung, zur Einheit, zum Ursprung, zu dem neuen Leben, das allein in Jesus ist. ${ }^{35}$ Dabei bleibt es nicht bei dem Ruf an sich, sondern der Mensch bekommt die Möglichkeit der Teilhabe an einem neuen Wirklichkeitsverständnis. Indem er immer mehr in das Bild Christi hineingestaltet wird, ${ }^{36}$ wird er Teil der einen Christuswirklichkeit. Der aner dipsychos, der "Mensch mit den zwei Seelen« (Jakobus 1,8), ${ }^{37}$ der von Selbst- und Gotteszweifel Zermarterte, wird ganz und gar auf den Einen, auf Christus hin verwiesen, in dem jeder Zweifel und jede Zwiespältigkeit überwunden ist.

\footnotetext{
- Dr. Christine Schliesser arbeitet an ihrer Habilitation zum Thema „Die Institutionalisierung des Ethikdiskurses« in Verbindung mit dem Ethik-Zentrum der Universität Zürich.
}

\footnotetext{
34 "Methodisch gesprochen müssen wir bei Bonhoeffer von `Christozentrik`sprechen«. E. Feil, Die Theologie Dietrich Bonhoeffers. Hermeneutik, Christologie, Weltverständnis, München ${ }^{4} 1991,139$, vgl. 215. "Regardless of what date is chosen during the twelve years of resistance, Bonhoeffer's theology and ethics are thoroughly Christological «, L.L. Rasmussen, Dietrich Bonhoeffer, Reality and Resistance, Nashville 1972, 89. "Bonhoeffer dachte von Anfang bis zum Ende christozentrisch und christokratisch.«, R. Mayer, Christuswirklichkeit. Grundlagen, Entwicklungen und Konsequenzen der Theologie Dietrich Bonhoeffers, Stuttgart 1969, 19.

35 Bonhoeffer, Ethik, 321.

${ }^{36}$ Vgl. das Kapitel "Das Bild Christi« in Bonhoeffers Nachfolge. Dietrich Bonhoeffer. Nachfolge, DBW 4, hg. v. M. Kuske und I. Tödt, München 1989, 297-304, hier 303: "Der menschgewordene, der gekreuzigte und der verklärte Christus nimmt Gestalt an in den Einzelnen".

${ }^{37}$ Bonhoeffer, Ethik, 321.
} 